\title{
ASYMPTOTIC NORMAL STRUCTURE AND FIXED POINTS OF NONEXPANSIVE MAPPINGS
}

\author{
J. B. BAILLON AND R. SCHÖNEBERG ${ }^{1}$
}

\begin{abstract}
A mapping $f$ defined on a subset $X$ of a Banach space $E$ and taking values in $E$ is said to be nonexpansive if $|f(x)-f(y)|<|x-y|$ for all $x, y \in X$. In this paper we introduce a promising new geometric property of Banach spaces and show that it yields via a minor modification of known arguments a new fixed point theorem for nonexpansive mappings which includes Kirk's famous result as well as a recent result of Karlovitz. We also discuss in detail a situation not covered by this result.
\end{abstract}

1. Statement of results. Throughout this paper, $E$ denotes a Banach space with norm || . If $X$ is a subset of $E$ and $f: X \rightarrow E$, then $f$ is said to be nonexpansive if $|f(x)-f(y)|<|x-y|$ for all $x, y \in X$. The space $E$ is said to have the BrowderGöhde-Kirk property (short: B-G-K property), if any nonexpansive self-mapping of a nonempty, closed, bounded and convex subset $X$ of $E$ has a fixed point in $X$.

In 1965, F. E. Browder [2] and D. Göhde [6] independently proved that every uniformly convex Banach space has the B-G-K property, while W. A. Kirk [8] established this property for the wider class of reflexive Banach spaces having normal structure. (Recall that $E$ is said to have normal structure, if for each bounded, closed and convex subset $X$ of $E$ consisting of more than one point there is a point $x \in X$ such that $\sup \{|y-x|: y \in X\}<\operatorname{diam}_{\mid}(X)(=\sup \{|u-v|:$ $u, v \in X\})$.)

Motivated by a certain method often employed in proofs of fixed point theorems for nonexpansive mappings (see Lemma 1 below), we introduce the following weakening of the concept of normal structure: A Banach space $E$ is said to have asymptotic normal structure, if for each bounded, closed and convex subset $X$ of $E$ consisting of more than one point and each sequence $\left\{x_{n}\right\}$ in $X$ satisfying $x_{n}-x_{n+1} \rightarrow 0$ as $n \rightarrow \infty$, there is a point $x \in X$ such that $\lim _{n \rightarrow \infty}\left|x_{n}-x\right|<$ $\operatorname{diam}_{1 \mid}(X)$.

THEOREM 1. Every reflexive Banach space with asymptotic normal structure has the $B-G-K$ property.

The interest in Theorem 1 stems, of course, from the fact that normal structure defines a narrower class of Banach spaces than asymptotic normal structure. This can be seen, among others, from our next result.

Received by the editors July 31, 1979 and, in revised form, April 15, 1980.

1980 Mathematics Subject Classification. Primary 47H10.

Key words and phrases. Nonexpansive mappings, normal structure, asymptotic normal structure.

${ }^{1}$ Part of this work was done while the second author was visiting the University of Iowa. 
Theorem 2. Let $\beta \geqslant 1$ and let $E_{\beta}$ be the real space $l_{2}$ renormed according to

$$
|x|_{\beta}=\max \left\{\|x\|_{2}, \beta\|x\|_{\infty}\right\}
$$

where $\|x\|_{\infty}$ denotes the $l_{\infty}$-norm and $\|x\|_{2}$ the $l_{2}$-norm. Then

(1) $E_{\beta}$ has normal structure if and only if $\beta<\sqrt{2}$, and

(2) $E_{\beta}$ has asymptotic normal structure if and only if $\beta<2$.

Theorem 2 has several most interesting consequences. On the one hand it establishes that Kirk's result is properly contained in our Theorem 1. On the other hand it yields (via Theorem 1) that for all $\beta<2, E_{\beta}$ has the B-G-K property, a fact first established by Karlovitz [7] (for the special case $\beta=\sqrt{2}$ ) using a rather elaborate though sophisticated method of proof. Finally, Theorem 2 illustrates the limits of the applicability of Theorem 1. To be more precise, it shows that Theorem 1 cannot be used to decide which of the spaces $E_{\beta}$ with $\beta>2$ have the B-G-K property. However, by using a certain "averaging procedure" we are able to establish the following fact.

THEOREM 3. The space $E_{2}$ has the $B-G-K$ property.

Unfortunately our arguments in the proof of Theorem 3 do not work in the setting $\beta>2$. And although one of the authors (J. B. Baillon) recently succeeded in establishing the B-G-K property for certain $E_{\beta}$ 's with $\beta>2$, this problem is in principle still open and a subject of further investigations.

2. Proofs. The following essentially well-known lemma will play the crucial role.

LEMMA 1. Let $E$ be a reflexive Banach space with norm ||, let $X \subset E$ be nonempty, closed, bounded and convex and let $f$ be a nonexpansive mapping of $X$ into $X$. Then

(1) there is a sequence $\left\{x_{n}\right\}$ in $X$ such that $x_{n}-f\left(x_{n}\right) \rightarrow 0$ and $x_{n}-x_{n+1} \rightarrow 0$ as $n \rightarrow \infty$.

(2) There is at least one nonempty, closed, bounded, convex and f-invariant subset $K$ of $X$ which is minimal with respect to $f$ (i.e., which contains no proper, nonempty, closed, bounded, convex and f-invariant subset).

(3) If $X$ is minimal with respect to $f$ and $\left\{x_{n}\right\}$ is a sequence in $X$ such that $x_{n}-f\left(x_{n}\right) \rightarrow 0$ as $n \rightarrow \infty$, then $\left|x_{n}-x\right| \rightarrow \operatorname{diam}_{||}(X)$ as $n \rightarrow \infty$ for all $x \in X$.

Proof. (1) Fix $z \in X$. Then Banach's contraction principle yields the existence of a sequence $\left\{x_{n}\right\}$ in $X$ satisfying $x_{n}=z / n+(1-1 / n) f\left(x_{n}\right)$. We have $x_{n}-f\left(x_{n}\right)$ $=\left(z-f\left(x_{n}\right)\right) / n \rightarrow 0$ as $n \rightarrow \infty$, and, by nonexpansiveness of $f$, for all $n \in N$,

$$
\begin{aligned}
\left|x_{n}-x_{n+1}\right| & =\left|\frac{1}{n(n+1)}\left(z-f\left(x_{n}\right)\right)+\left(1-\frac{1}{n+1}\right)\left(f\left(x_{n}\right)-f\left(x_{n+1}\right)\right)\right| \\
& \leqslant \frac{1}{n(n+1)}\left|z-f\left(x_{n}\right)\right|+\left(1-\frac{1}{n+1}\right)\left|x_{n}-x_{n+1}\right|,
\end{aligned}
$$

so that $\left|x_{n}-x_{n+1}\right|<\left|z-f\left(x_{n}\right)\right| / n \rightarrow 0$ as $n \rightarrow \infty$.

(2) Standard Zorn's lemma argument using reflexivity of $E$.

(3) See, e.g., [5] and [7]. 
REMARK. From many instances we got the impression that the problem of giving complete references to part (3) of this lemma seems to be very hard to solve. Since we are mostly concerned about mathematics we therefore decided to leave it without a solution.

Using Lemma 1 the proof of Theorem 1 is now straightforward and hence omitted. But for the proofs of Theorems 2 and 3 we need in addition the following results about the space $l_{2}$.

Lemma 2. Let $\beta>1, u, v, w \in E_{\beta}$ and $t \in[0,1]$. Then

$$
\|u-((1-t) v+t w)\|_{2}^{2}+t(1-t)\|v-w\|_{2}^{2}=(1-t)\|u-v\|_{2}^{2}+t\|u-w\|_{2}^{2} \text {. }
$$

Proof. \|\|$_{2}$ is a Hilbert-space-norm.

Lemma 3 (CF. EdelsteIn [3], [4]). Let $\beta>1$, let $X \subset E_{\beta}$ be closed, bounded and convex and let $\left\{x_{n}\right\}$ be a sequence in $X$. Then there exists a unique point $z \in X$ (called the " \|\|$_{2}$-asymptotic-center of $\left\{x_{n}\right\}$ in $X$ ") which minimizes the functional $x \mapsto \lim \sup _{n \rightarrow \infty}\left\|x_{n}-x\right\|_{2}$. This point $z$ satisfies, in addition,

(a) $\lim \sup _{n \rightarrow \infty}\left\|x_{n}-z\right\|_{2}^{2}+\|z-x\|_{2}^{2}<\lim \sup _{n \rightarrow \infty}\left\|x_{n}-x\right\|_{2}^{2}$ for all $x \in X$, and

(b) $2 \lim \sup _{n \rightarrow \infty}\left\|x_{n}-z\right\|_{2}^{2} \leqslant \lim \sup _{p \rightarrow \infty}\left\{\lim \sup _{n \rightarrow \infty}\left\|x_{n}-x_{p}\right\|_{2}^{2}\right\}$.

Proof. The functional $g(x)=\lim \sup _{n \rightarrow \infty}\left\|x_{n}-x\right\|_{2}$ is continuous and convex and satisfies, by Lemma 2 ,

$$
g\left(\frac{1}{2}\left(y_{1}+y_{2}\right)\right)^{2}+\frac{1}{4}\left\|y_{1}-y_{2}\right\|_{2}^{2}<\frac{1}{2} g\left(y_{1}\right)^{2}+\frac{1}{2} g\left(y_{2}\right)^{2}
$$

for all $y_{1}, y_{2} \in X$. Hence $g$ has a unique minimizer in $X$.

(a) For $t \in(0,1)$, Lemma 2 yields

$$
\begin{aligned}
g(z)^{2}+t(1-t)\|x-z\|_{2}^{2} & <g((1-t) x+t z)^{2}+t(1-t)\|x-z\|_{2}^{2} \\
& <(1-t) g(x)^{2}+t g(z)^{2},
\end{aligned}
$$

so that $g(z)^{2}+t\|x-z\|_{2}^{2}<g(x)^{2}$.

(b) $x=x_{p}$ in (a).

LEMMA 4. Let $1 \leqslant \beta<2$, let $X \subset E_{\beta}$ be closed, bounded and convex with $d=\operatorname{diam}_{||_{\beta}}(X)$, let $\left\{x_{n}\right\}$ be a sequence in $X$ such that $x_{n}-x_{n+1} \rightarrow 0$ as $n \rightarrow \infty$ and $\left|x_{n}-x\right|_{\beta} \rightarrow d$ as $n \rightarrow \infty$ for all $x \in X$, and let $z \in X$ denote the \|\|$_{2}$-asymptoticcenter of $\left\{x_{n}\right\}$ in $X$. Then $\lim \sup _{n \rightarrow \infty}\left\|x_{n}-z\right\|_{2}^{2}>2(d / \beta)^{2}$.

Proof. Assume, for a contradiction, that $\lim _{\sup _{n \rightarrow \infty}}\left\|x_{n}-z\right\|_{2}^{2}<2(d / \beta)^{2}$ (and hence $d>0$ ). Since, by Lemma 3(b),

$$
\limsup _{n \rightarrow \infty}\left\|x_{n}-z\right\|_{2}^{2}<\frac{d^{2}}{2}
$$

and $\left|x_{n}-z\right|_{\beta} \rightarrow d$ as $n \rightarrow \infty$, the definition of ||$_{\beta}$ implies $\left\|x_{n}-z\right\|_{\infty} \rightarrow d / \beta$ as $n \rightarrow \infty$. Hence there is $r<2(d / \beta)^{2}$ and $n_{0} \in \mathbf{N}$ such that for $n>n_{0}:\left\|x_{n}-z\right\|_{2}^{2}<$ $r,\left\|x_{n}-x_{n+1}\right\|_{\infty}<\left\|x_{n+1}-z\right\|_{\infty}$ and

$$
\left\|x_{n}-z\right\|_{\infty}^{2}+\left(\left\|x_{n+1}-z\right\|_{\infty}-\left\|x_{n}-x_{n+1}\right\|_{\infty}\right)^{2}>r \text {. }
$$


For $n \in \mathbf{N}$ select $j_{n} \in \mathbf{N}$ such that $\left\|x_{n}-z\right\|_{\infty}=\left|x_{n}\left(j_{n}\right)-z\left(j_{n}\right)\right|\left(x_{n}, z \in l_{2} !\right)$ and set $k=j_{n_{0}}$.

ClaIm 1. $j_{n}=k$ for $n \geqslant n_{0}$. If this is not true, there is $n>n_{0}$ with $j_{n} \neq j_{n+1}$. Then

$$
\begin{aligned}
r & >\left\|x_{n}-z\right\|_{2}^{2} \\
& >\left|x_{n}\left(j_{n}\right)-z\left(j_{n}\right)\right|^{2}+\left|x_{n}\left(j_{n+1}\right)-z\left(j_{n+1}\right)\right|^{2} \\
& >\left\|x_{n}-z\right\|_{\infty}^{2}+\left(\left\|x_{n+1}-z\right\|_{\infty}-\left\|x_{n}-x_{n+1}\right\|_{\infty}\right)^{2} \\
& >r,
\end{aligned}
$$

a contradiction.

Claim 2. If $x \in X$ is a weak limit point of $\left\{x_{n}\right\}$, then $\|x-z\|_{2}^{2}+d^{2} / \beta^{2}<$ $d^{2} / 2$.

Suppose $x_{n_{j}} \rightarrow x$ as $j \rightarrow \infty$. Then, for $j \in \mathbf{N}$,

$$
\left\|x_{n,}-z\right\|_{2}^{2}=\left\|x_{n_{j}}-x\right\|_{2}^{2}+2\left\langle x_{n,}-x, x-z\right\rangle+\|x-z\|_{2}^{2},
$$

so that by (*)

$$
\limsup _{j \rightarrow \infty}\left\|x_{n_{j}}-x\right\|_{2}^{2}+\|x-z\|_{2}^{2}<\limsup _{j \rightarrow \infty}\left\|x_{n_{j}}-z\right\|_{2}^{2}<\frac{d^{2}}{2} .
$$

Since $\left\|x_{n_{j}}-x\right\|_{2} \geqslant\left|x_{n_{j}}-x\right|_{\beta} / \beta \rightarrow d / \beta$ as $j \rightarrow \infty$, this inequality gives the desired conclusion.

We now complete the proof of Lemma 4 . Let $x$ be any weak limit point of $\left\{x_{n}\right\}$. Then Claims 1 and 2 imply

$$
\begin{aligned}
\frac{d^{2}}{\beta^{2}} & =\lim _{n \rightarrow \infty}\left\|x_{n}-z\right\|_{\infty}^{2}=\lim _{n \rightarrow \infty}\left|x_{n}(k)-z(k)\right|^{2} \\
& =|x(k)-z(k)|^{2}<\|x-z\|_{2}^{2}<\frac{d^{2}}{2}-\frac{d^{2}}{\beta^{2}} .
\end{aligned}
$$

Hence $\beta=2$ and $|x(k)-z(k)|=\|x-z\|_{2}=d / 2$, so that $x(j)=z(j)$ for $j \neq k$ and $|x(k)-z(k)|=d / 2$. But the real sequence $\left\{x_{n}(k)\right\}$ satisfies $x_{n}(k)-x_{n+1}(k)$ $\rightarrow 0$ as $n \rightarrow \infty$, so that the set of limit points of $\left\{x_{n}(k)\right\}$ is an interval. This and the above imply that $\left\{x_{n}\right\}$ converges weakly and the weak limit point of $\left\{x_{n}\right\}$ is, of course, the \|\|$_{2}$-asymptotic-center $z$ (cf. proof of Claim 2), yielding $0=\mid z(k)-$ $z(k) \mid=d / 2$, i.e., $d=0$, which contradicts our assumption.

Proof of Theorem 2. (1) If $\beta>\sqrt{2}$, the subset $X=\left\{x \in E_{\beta}:\|x\|_{2}<1\right.$ and $x(j)>0$ for all $j\}$ of $E_{\beta}$ is easily seen to be bounded, closed and convex, to consist of more than one point and to have the property that $\sup \left\{|y-x|_{\beta}: y \in X\right\}=\beta$ $=\operatorname{diam}_{||_{\beta}}(X)$ for all $x \in X$. Suppose now that $\beta<\sqrt{2}$ and let $X$ be a closed, bounded and convex subset of $E_{\beta}$ with $d=\operatorname{diam}_{||_{\beta}}(X)>0$. Then a slight modification of the proof of Lemma 3 yields existence of a unique point $z \in X$ which minimizes the functional $x \mapsto \sup \left\{\|y-x\|_{2}: y \in X\right\}$ and satisfies, in addition, $\sup \left\{\|y-z\|_{2}: y \in X\right\}<\operatorname{diam}_{\|\|_{2}}(X) / \sqrt{2}$. But then we have for $y \in X$ :

$$
|y-z|_{\beta} \leqslant \beta\|y-z\|_{2}<\frac{\beta}{\sqrt{2}} \operatorname{diam}_{\|\|_{2}}(X)<\frac{\beta}{\sqrt{2}} d<d,
$$

thus establishing the second part of assertion (1). 
(2) If $\beta>2$, we define $X \subset E_{\beta}$ by $X=\left\{x \in E_{\beta}:|x|_{\sqrt{2}}<\sqrt{2}\right.$ and $x(j)>0$ for all $j\}$ and a sequence $\left\{x_{n}\right\}$ in $X$ by

$$
x_{n}=\frac{(2 k+1)^{2}-n}{4 k+1} e_{k}+e_{k+1} \text { if }(2 k)^{2}<n<(2 k+1)^{2}
$$

and

$$
x_{n}=e_{k+1}+\frac{n-(2 k+1)^{2}}{4 k+3} e_{k+2} \text { if }(2 k+1)^{2}<n<(2 k+2)^{2},
$$

where $\left\{e_{k}\right\}$ denotes the usual orthonormal basis of $l_{2}$. Then some straightforward reasonings yield that $X$ is closed, bounded and convex with $\operatorname{diam}_{\left.1\right|_{\beta}}(X)=\beta$ and that $x_{n}-x_{n+1} \rightarrow 0$ as $n \rightarrow \infty$, while $\left|x_{n}-x\right|_{\beta} \rightarrow \beta$ as $n \rightarrow \infty$ for all $x \in X$. Therefore $E_{\beta}$ does not have asymptotic normal structure. Suppose now that $\beta<2$, let $X \subset E_{\beta}$ be closed, bounded and convex with $d=\operatorname{diam}_{\left.1\right|_{\beta}}(X)>0$, and let $\left\{x_{n}\right\}$ be a sequence in $X$ such that $x_{n}-x_{n+1} \rightarrow 0$ as $n \rightarrow \infty$. By Lemma 3(b) we have (letting $z$ denote the \|\|$_{2}$-asymptotic-center of $\left\{x_{n}\right\}$ in $X$ ): $\lim \sup _{n \rightarrow \infty}\left\|x_{n}-z\right\|_{2}^{2}<$ $d^{2} / 2<2(d / \beta)^{2}$, so that Lemma 4 yields existence of a point $x \in X$ such that $\lim \inf _{n \rightarrow \infty}\left|x_{n}-x\right|_{\beta}<d$.

The proof of Theorem 3 requires further information about $l_{2}$.

Lemma 5. Let $X \subset E_{2}$ be closed, bounded and convex and let $\left\{y_{n}\right\}$ be a sequence in $X$ such that $\left|y_{n}-x\right|_{2} \rightarrow d=\operatorname{diam}_{1_{2}}(X)$ as $n \rightarrow \infty$ for all $x \in X$. Then $\left\|y_{n}-x\right\|_{\infty} \rightarrow d / 2$ as $n \rightarrow \infty$ for all $x \in X$.

Proof. By definition of $\|\left.\right|_{\beta}$, we have $2\left\|y_{n}-x\right\|_{\infty}<\left|y_{n}-x\right|_{2}<d$ for all $n \in \mathbf{N}$, $x \in X$. Assume now, for a contradiction, that there exist $\varepsilon>0$, a subsequence $\left\{y_{n_{k}}\right\}$ of $\left\{y_{n}\right\}$ and $x \in X$ such that $\left\|y_{n_{k}}-x\right\|_{\infty}<d / 2-\varepsilon$ for all $k$. If $z \in X$ denotes the \|\|$_{2}$-asymptotic-center of $\left\{y_{n}\right\}$ in $X$, we get for $k \in \mathbf{N}$

$$
\left\|y_{n_{k}}-\frac{1}{2}(x+z)\right\|_{\infty}<\frac{1}{2}\left\|y_{n_{k}}-x\right\|_{\infty}+\frac{1}{2}\left\|y_{n_{k}}-z\right\|_{\infty}<(d-\varepsilon) / 2
$$

and (by Lemma 2)

$$
\begin{aligned}
\left\|y_{n_{k}}-\frac{1}{2}(x+z)\right\|_{2}^{2} & <\frac{1}{2}\left\|y_{n_{k}}-x\right\|_{2}^{2}+\frac{1}{2}\left\|y_{n_{k}}-z\right\|_{2}^{2} \\
& <\frac{1}{2} d^{2}+\frac{1}{2}\left\|y_{n_{k}}-z\right\|_{2}^{2},
\end{aligned}
$$

which, together with Lemma 3(b), yields $\lim \sup _{k \rightarrow \infty}\left\|y_{m_{k}}-\frac{1}{2}(x+z)\right\|_{2}^{2}<\frac{3}{4} d^{2}$. Therefore

$$
\underset{k \rightarrow \infty}{\lim \sup }\left|y_{n_{k}}-\frac{1}{2}(x+z)\right|_{2}<\max \left\{\sqrt{\frac{3}{4}} d, d-\varepsilon\right\}
$$

which contradicts our assumption that $\left|y_{n}-\frac{1}{2}(x+z)\right|_{2} \rightarrow d$ as $n \rightarrow \infty$.

LEMMA 6. Let $X \subset E_{2}$ be closed, bounded and convex with $d=\operatorname{diam}_{1_{2}}(X)$, let $f$ : $X \rightarrow X$ be nonexpansive and let $\left\{u_{k}\right\}$ and $\left\{v_{k}\right\}$ be sequences in $X$ such that $u_{k}-f\left(u_{k}\right) \rightarrow 0$ and $v_{k}-f\left(v_{k}\right) \rightarrow 0$ as $k \rightarrow \infty$ and

$$
\lim _{k \rightarrow \infty}\left\{\lim _{p \rightarrow \infty}\left\|u_{k}-v_{p}\right\|_{2}^{2}\right\}=d^{2} \text {. }
$$


Suppose furthermore that $X$ is minimal with respect to $f$. Then

$$
\limsup _{k \rightarrow \infty}\left\{\limsup _{p \rightarrow \infty}\left\|\frac{1}{2}\left(u_{k}+v_{p}\right)-x\right\|_{\infty}\right\}=\frac{d}{2} \quad \text { for all } x \in X .
$$

Proof. For $k, p \in \mathrm{N}$, we have (by Lemma 2)

$$
\begin{aligned}
\| f\left(\frac{1}{2}\left(u_{k}+v_{p}\right)\right)-\frac{1}{2}\left(u_{k}+\right. & \left.v_{p}\right)\left\|_{2}^{2}+\frac{1}{4}\right\| u_{k}-v_{p} \|_{2}^{2} \\
& =\frac{1}{2}\left\|f\left(\frac{1}{2}\left(u_{k}+v_{p}\right)\right)-u_{k}\right\|_{2}^{2}+\frac{1}{2}\left\|f\left(\frac{1}{2}\left(u_{k}+v_{p}\right)\right)-v_{p}\right\|_{2}^{2} .
\end{aligned}
$$

Since, by nonexpansiveness of $f$,

and (similarily)

$$
\begin{aligned}
\left\|f\left(\frac{1}{2}\left(u_{k}+v_{p}\right)\right)-u_{k}\right\|_{2} & <\left|f\left(\frac{1}{2}\left(u_{k}+v_{p}\right)\right)-u_{k}\right|_{2} \\
& <\frac{1}{2}\left|v_{p}-u_{k}\right|_{2}+\left|f\left(u_{k}\right)-u_{k}\right|_{2} \\
& <d / 2+\left|f\left(u_{k}\right)-u_{k}\right|_{2},
\end{aligned}
$$

$$
\left\|f\left(\frac{1}{2}\left(u_{k}+v_{p}\right)\right)-v_{p}\right\|_{2}<d / 2+\left|f\left(v_{p}\right)-v_{p}\right|_{2},
$$

the above inequality together with our assumptions implies

$$
\underset{k \rightarrow \infty}{\limsup }\left\{\limsup _{p \rightarrow \infty}\left\|f\left(\frac{1}{2}\left(u_{k}+v_{p}\right)\right)-\frac{1}{2}\left(u_{k}+v_{p}\right)\right\|_{2}^{2}\right\}=0,
$$

so that

$$
\limsup _{k \rightarrow \infty}\left\{\limsup _{p \rightarrow \infty}\left|f\left(\frac{1}{2}\left(u_{k}+v_{p}\right)\right)-\frac{1}{2}\left(u_{k}+v_{p}\right)\right|_{2}\right\}=0 .
$$

Assume now existence of $x \in X$ with

$$
\limsup _{k \rightarrow \infty}\left\{\limsup _{p \rightarrow \infty}\left\|\frac{1}{2}\left(u_{k}+v_{p}\right)-x\right\|_{\infty}\right\}<r<\frac{d}{2} \text {. }
$$

Then, by $(+)$ and $(++)$, we can find subsequences $\left\{u_{k_{q}}\right\}$ and $\left\{v_{p_{q}}\right\}$ such that for $q \in \mathbf{N}:\left|f\left(y_{q}\right)-y_{q}\right|_{2}<2 / q$ and $\left\|y_{q}-x\right\|_{\infty}<r$, where $y_{q}=\frac{1}{2}\left(u_{k_{q}}+v_{p_{q}}\right)$. Then $\lim _{q \rightarrow \infty}\left|f\left(y_{q}\right)-y_{q}\right|_{2}=0$ and $\lim \sup _{q \rightarrow \infty}\left\|y_{q}-x\right\|_{\infty}<r<d / 2$, which contradicts Lemma 5 by Lemma 1(3).

LEMMA 7. Let $\left\{u_{k}\right\},\left\{v_{k}\right\} \subset l_{2}$ and $d>0$ such that $v_{k} \rightarrow 0$ as $k \rightarrow \infty,\left\|v_{k}\right\|_{\infty} \rightarrow$ $d / 2$ and $\left\|u_{k}\right\|_{\infty} \rightarrow d / 2$ as $k \rightarrow \infty,\left\|u_{k}-v_{p}\right\|_{\infty}<d / 2$ for all $k, p$ and

$$
\underset{k \rightarrow \infty}{\lim \sup }\left\{\lim _{p \rightarrow \infty} \sup _{k}+u_{p} \|_{2}^{2}\right\}=d^{2} \text {. }
$$

Then

$$
\limsup _{k \rightarrow \infty}\left\{\limsup _{p \rightarrow \infty}\left\|u_{k}+v_{p}\right\|_{\infty}\right\}<d .
$$

Proof. Let $\varepsilon>0$ such that $d^{2} / 4+(d+\varepsilon)^{2}<\frac{3}{2}(d-\varepsilon)^{2}$. There is $k_{0} \in \mathbf{N}$ such that $\lim \sup _{p \rightarrow \infty}\left\|u_{k}+v_{p}\right\|_{2}^{2}<(d+\varepsilon)^{2}$ and $\left\|u_{k}\right\|_{\infty}>(d-\varepsilon) / 2$ for $k>k_{0}$. We claim $\lim \sup _{p \rightarrow \infty}\left\|u_{k}+v_{p}\right\|_{\infty}\left\langle d-\varepsilon\right.$ for all $k>k_{0}$. If this is not true, there is $k>k_{0}$ such that $\lim \sup _{p \rightarrow \infty}\left\|u_{k}+v_{p}\right\|_{\infty}>d-\varepsilon$. Select $n \in \mathrm{N}$ such that $\left|u_{k}(n)\right|=$ $\left\|u_{k}\right\|_{\infty}(\geqslant(d-\varepsilon) / 2)$. Since $v_{p} \rightarrow 0$ and $\left\|v_{p}\right\|_{\infty} \rightarrow d / 2$ as $p \rightarrow \infty$, we can find 
$p, m \in \mathbf{N}$ such that $\left\|u_{k}+v_{p}\right\|_{\infty}>d-\varepsilon,\left\|u_{k}+v_{p}\right\|_{2}^{2}<(d+\varepsilon)^{2},\left|v_{p}(m)\right|=\left\|v_{p}\right\|_{\infty}$ $>(d-\varepsilon) / 2$ and $m \neq n$. For $j \in \mathbf{N}$, we have

$$
\begin{aligned}
\left|u_{k}(j)+v_{p}(j)\right|^{2} & =2\left|u_{k}(j)\right|^{2}+2\left|v_{p}(j)\right|^{2}-\left|u_{k}(j)-v_{p}(j)\right|^{2} \\
& \geqslant 2\left|u_{k}(j)\right|^{2}+2\left|v_{p}(j)\right|^{2}-d^{2} / 4
\end{aligned}
$$

Hence

$$
\left|u_{k}(n)+v_{p}(n)\right|^{2}>2\left(\frac{d-\varepsilon}{2}\right)^{2}-\frac{d^{2}}{4}
$$

and

$$
\left|u_{k}(m)+v_{p}(m)\right|^{2}>2\left(\frac{d-\varepsilon}{2}\right)^{2}-\frac{d^{2}}{4} .
$$

Now select $i \in \mathbf{N}$ with $\left|u_{k}(i)+v_{p}(i)\right|=\left\|u_{k}+v_{p}\right\|_{\infty}(>d-\varepsilon)$. Then, since $\{i\} \neq$ $\{n, m\}$, the above inequalities yield

$$
\begin{aligned}
(d+\varepsilon)^{2} & >\left\|u_{k}+v_{p}\right\|_{2}^{2}>(d-\varepsilon)^{2}+2\left(\frac{d-\varepsilon}{2}\right)^{2}-\frac{d^{2}}{4} \\
& =\frac{3}{2}(d-\varepsilon)^{2}-\frac{d^{2}}{4}>(d+\varepsilon)^{2}
\end{aligned}
$$

a contradiction.

Proof of Theorem 3. Let $X$ be a nonempty, closed, bounded and convex subset of $E_{2}$ and let $f$ be a nonexpansive self-mapping of $X$. We may suppose that $X$ is minimal with respect to $f($ Lemma $1(2))$. Let $d=\operatorname{diam}_{\left.\right|_{1}}(X)$ and assume, for a contradiction, that $d>0$. By Lemma 1(1) there is a sequence $\left\{x_{n}\right\}$ in $X$ with $x_{n}-f\left(x_{n}\right) \rightarrow 0$ and $x_{n}-x_{n+1} \rightarrow 0$ as $n \rightarrow \infty$. Let $z \in X$ denote the \|\|$_{2}$-asymptotic-center of $\left\{x_{n}\right\}$ in $X$. By Lemma 1(3) and Lemma 4, we have

(a) $\lim \sup _{n \rightarrow \infty}\left\|x_{n}-z\right\|_{2}^{2}>d^{2} / 2$.

Select a subsequence $\left\{x_{n_{k}}\right\}$ of $\left\{x_{n}\right\}$ with $x_{n_{k}} \rightarrow x \in X$ and $\left\|x_{n_{k}}-z\right\|_{2}^{2} \rightarrow$ $\lim \sup _{n \rightarrow \infty}\left\|x_{n}-z\right\|_{2}^{2}$ as $k \rightarrow \infty$ and then a subsequence $\left\{x_{m_{k}}\right\}$ of $\left\{x_{n}\right\}$ with $x_{m_{k}} \rightarrow y \in X$ and $\left\|x_{m_{k}}-x\right\|_{2}^{2} \rightarrow \lim \sup _{n \rightarrow \infty}\left\|x_{n}-x\right\|_{2}^{2}$ as $k \rightarrow \infty$. Then (a) and Lemma 3(a) yield

$$
\begin{aligned}
d^{2} & >\lim _{k \rightarrow \infty}\left\{\lim _{p \rightarrow \infty}\left\|x_{n_{k}}-x_{m_{p}}\right\|_{2}^{2}\right\} \\
& =\underset{n \rightarrow \infty}{\lim \sup _{n}}\left\|x_{n}-x\right\|_{2}^{2}+\underset{n \rightarrow \infty}{\lim \sup }\left\|x_{n}-z\right\|_{2}^{2}-\|z-x\|_{2}^{2} \\
& >2 \underset{n \rightarrow \infty}{\lim \sup }\left\|x_{n}-z\right\|_{2}^{2}>d^{2},
\end{aligned}
$$

so that

(b) $\lim \sup _{n \rightarrow \infty}\left\|x_{n}-z\right\|_{2}^{2}=d^{2} / 2$, and

(c) $\lim _{k \rightarrow \infty}\left\{\lim _{p \rightarrow \infty}\left\|x_{n_{k}}-x_{m_{p}}\right\|_{2}^{2}\right\}=d^{2}$.

Hence Lemma 6 (with $u_{k}=x_{n_{k}}$ and $v_{k}=x_{m_{k}}$ ) implies

(d) $\lim \sup _{k \rightarrow \infty}\left\{\lim \sup _{p \rightarrow \infty}\left\|\frac{1}{2}\left(x_{n_{k}}+x_{m_{p}}\right)-u\right\|_{\infty}\right\}=d / 2$ for all $u \in X$. 
This yields, in particular,

$$
\underset{k \rightarrow \infty}{\limsup }\left\{\limsup _{p \rightarrow \infty}\left\|\frac{1}{2}\left(x_{n_{k}}+x_{m_{p}}\right)-\frac{1}{2}(x+y)\right\|_{2}^{2}\right\}>\frac{d^{2}}{4},
$$

so that (using (b))

$$
\begin{aligned}
\frac{d^{2}}{4} & \leqslant \limsup _{k \rightarrow \infty}\left\{\limsup _{p \rightarrow \infty}\left\|\frac{1}{2}\left(x_{n_{k}}+x_{m_{p}}\right)-\frac{1}{2}(x+y)\right\|_{2}^{2}\right\} \\
& =\frac{1}{4} \lim _{k \rightarrow \infty}\left\|x_{n_{k}}-x\right\|_{2}^{2}+\frac{1}{4} \lim _{p \rightarrow \infty}\left\|x_{m_{p}}-y\right\|_{2}^{2} \\
& =\frac{1}{4}\left(\frac{d^{2}}{2}-\|z-x\|_{2}^{2}\right)+\frac{1}{4}\left(\frac{d^{2}}{2}-\|z-y\|_{2}^{2}\right) \\
& =\frac{d^{2}}{4}-\frac{1}{4}\left(\|z-x\|_{2}^{2}+\|z-y\|_{2}^{2}\right)
\end{aligned}
$$

proving $x=y=z$. Let now $u_{k}=x_{n_{k}}-z$ and $v_{k}=x_{m_{k}}-z$. Then we have

(e) $v_{k} \rightarrow 0$ as $k \rightarrow \infty$,

(f) $\left\|v_{k}\right\|_{\infty} \rightarrow d / 2$ and $\left\|u_{k}\right\|_{\infty} \rightarrow d / 2$ as $k \rightarrow \infty$ (by Lemma 1(3) and Lemma 5),

(g) $\left\|u_{k}-v_{p}\right\|_{\infty} \leqslant d / 2$ for all $k, p \in \mathbf{N}$ (by definition of $d$ ),

(h) $\lim _{k \rightarrow \infty}\left\{\lim _{p \rightarrow \infty}\left\|u_{k}-v_{p}\right\|_{2}^{2}\right\}=d^{2}$ (by (c)) and

(i) $\lim \sup _{k \rightarrow \infty}\left\{\lim \sup _{p \rightarrow \infty}\left\|u_{k}+v_{p}\right\|_{\infty}\right\}=d$ (by (d)). Since for all $k, p$

$$
\left\|u_{k}+v_{p}\right\|_{2}^{2}=2\left\|u_{k}\right\|_{2}^{2}+2\left\|v_{p}\right\|_{2}^{2}-\left\|u_{k}-v_{p}\right\|_{2}^{2}
$$

we also have

(j) $\lim _{k \rightarrow \infty}\left\{\lim _{p \rightarrow \infty}\left\|u_{k}+v_{p}\right\|_{2}^{2}\right\}=d^{2}$, which is impossible by Lemma 7 .

3. Acknowledgement. We are grateful to W. A. Kirk for many most valuable discussions and to W. L. Bynum [1], T. Kuczumov (private communication) and W. Ray (private communication) for providing us with examples of spaces lacking asymptotic normal structure.

\section{REFERENCES}

1. W. L. Bynum, An example of a reflexive Banach space lacking asymptotic normal structure (preprint).

2. F. E. Browder, Nonexpansive nonlinear operators in Banach space, Proc. Nat. Acad. Sci. U.S.A. 54 (1965), 1041-1044.

3. M. Edelstein, The construction of an asymptotic center with a fixed-point property, Bull. Amer. Math. Soc. 78 (1972), 206-208.

4. __ Fixed point theorems in uniformly convex Banach spaces, Proc. Amer. Math. Soc. 44 (1974), 369-374.

5. K. Goebel, On the structure of minimal invariant sets for nonexpansive mappings, Ann. Univ. Mariae Curie-Skłodowska Sect. A.

6. D. Göhde, Zum Prinzip der kontraktiven Abbildung, Math. Nachr. 30 (1965), 251-258.

7. L. A. Karlovitz, Existence of fixed points of nonexpansive mappings in a space without normal structure, Pacific J. Math. 66 (1976), 153-158.

8. W. A. Kirk, A fixed point theorem for mappings which do not increase distance, Amer. Math. Monthly 72 (1965), 1004-1006.

École Polytechnque, Centre de Mathímatiques, Plateau de Palaiseau, 91128 Palaiseau Cedex, France

OSdorfer Landstrasse 311, 2000 Hamburg 55, West Germany 